Изв. Крымск. Астрофиз. Обсерв. 117, № 1, 56-61 (2021) doi:10.31059/izcrao-vol117-iss1-pp56-61

УДК 524.3-14 + 524.33-56

\title{
Поиск циклов активности у избранных F-, G- и K-карликов по многолетним широкоугольным фотометрическим наблюдениям
}

\author{
А.А. Шляпников, Н.И. Бондарь, М.А. Горбунов \\ ФГБУН "Крымская астрофизическая обсерватория РАН", Научный, 298409, Крым \\ aas@craocrimea.ru
}

Поступила в редакцию 5 ноября 2021 г.

Аннотация. Рассмотрены возможности использования данных проекта наземных панорамных наблюдений KWS для поиска циклов активности у ярких F - K карликов. Приведены способы и результаты обработки наблюдательного материала, описано формирование интерактивной базы данных.

Ключевые слова: звезды солнечного типа, красные карлики, циклы активности, базы данных

\section{1 Введение}

В основе проявления различных типов активности звезд средних и малых масс спектральных типов от F до L, таких как темные пятна и светлые факелы фотосферы, яркие активные области хромосферы, вспышки в различных спектральных диапазонах, выбросы коронального вещества, лежит изменение структуры магнитных полей. Магнитные поля являются источником энергии этой активности и в зависимости от их конфигурации, генерации и диссипации в атмосфере звезды развиваются неоднородности разных масштабов, характеристики которых меняются на временных интервалах от долей секунд до десятилетий.

Для поиска и изучения циклов активности, обусловленных развитием фотосферных пятен, необходимы многолетние фотометрические ряды. В настоящее время получить такие ряды можно при совместном рассмотрении результатов электрофотометрии и данных широкоугольных фотометрических обзоров, которые проводятся с 2000 г. Анализ долговременных вариаций блеска по данным наземных и космических наблюдений, выполненный для OU Gem, V1005 Ori и AU Mic, показал, что у этих звезд можно заподозрить проявление циклов длительностью около 40 лет (Bondar', Katsova, 2020). У всех этих звезд есть циклы 10-16 лет, а у EQ Vir, V1005 Ori и AU Міс наблюдаются также короткие циклы длительностью 5-6 лет. Во всех случаях обнаруженной циклической активности амплитуда переменности на многолетних интервалах наблюдений составляла в среднем не более 0.2 звездной величины, а для коротких циклов $-0^{\mathrm{m}} .04-0^{\mathrm{m}} .08$. Более значительные изменения блеска $\left(\sim 0^{\mathrm{m}} .8\right)$ были обнаружены по результатам анализа вековых изменений среднегодовых $V$-величин у звезды V833 Tau за 120 лет (с 1899 по 2019 год) - самого продолжительного ряда фотометрических наблюдений (Stepanov et al., 2020).

В настоящей работе, продолжая ранее начатые исследования по поиску циклов изменения блеска у звезд нижней части главной последовательности (Bondar' et al., 2019), рассматривается возможность использования данных фотометрии из базы KWS (Kyoto Wide-field Survey) для F-K карликов 4-9 звездной величины. Приведено описание разработанного интерактивного приложения с информацией об исследуемых объектах, рассматривается повышение точности входных данных при первичной обработке, использование фильтрации для выявления случайных импульсных событий повышения или понижения блеска, что важно для обнаружения малоамплитудных изменений блеска звезды. Для ряда звезд приведены результаты поиска циклов на 10-летних интервалах. 


\section{2 Интерактивное приложение с данными для анализа}

Специальное интерактивное приложение было создано для работы с данными об исследуемых объектах (рис. 1). Оно включает сетевой доступ к основной информации о звездах в базах данных SIMBAD (Wenger et al., 2000) и KWS (Maehara, 2014). Также на странице размещена информация об обнаруженных или заподозренных циклах активности, ссылки на иллюстрации к отфильтрованным и усредненным по годам наблюдений кривым блеска, в том числе и к данным, по которым они построены. Страница обновляется по мере накопления новой информации.

\begin{tabular}{|c|c|c|c|c|c|c|c|c|c|c|c|c|c|c|}
\hline \multicolumn{15}{|c|}{$\begin{array}{l}\text { The search for a possible cyclic variability } \\
\text { from KWS data in selected F - K dwarfs } \\
\text { Website is under construction. Test version. Contact (magecrao.ru, aas(erao.ru). } \\
\text { n is presented on the search for possible cyclic variability of selected stars from the list of objects [1, 2]. }\end{array}$} \\
\hline $\begin{array}{r}\text { Tab. } \\
1 . \\
2 . \\
3 . \\
4 . \\
5 . \\
6 . \\
7 . \\
8 . \\
9 . \\
10 . \\
11 . \\
12 . \\
13 . \\
14 .\end{array}$ & $\begin{array}{l}1 \text { le descript } \\
\text { H } \\
\text { RAJ (2000. } \\
\text { DEJ (2000. } \\
\text { S IMBAD } \\
\text { v mag } \\
\text { Sp_Type } \\
\text { P_rot } \\
\text { KWS } \\
\text { Cycle-1 } \\
\text { CYcle-2 } \\
\text { LC (KWS) } \\
\text { LC (FBD) } \\
\text { LC (YED) } \\
\text { Hote. }\end{array}$ & $\begin{array}{l}\text { ion: } \\
\text { o) } \\
\text { 0) } \\
\text { 0) } \\
- \\
- \\
- \\
- \\
- \\
- \\
- \\
- \\
- \\
- \\
-\end{array}$ & $\begin{array}{l}\text { record } r \\
\text { barycent } \\
\text { barycent } \\
\text { link to } \\
\text { v-magnit } \\
\text { spectral } \\
\text { rotation } \\
\text { link to } \\
\text { cycle } 1 \\
\text { cycle } 2 \\
\text { link to } \\
\text { link to } \\
\text { link to }\end{array}$ & $\begin{array}{l}\text { number. } \\
\text { tric right } \\
\text { tric declin } \\
\text { the SIMBAD } \\
\text { tude from } \mathrm{s} \\
\text { type from } \\
\text { n period (d. } \\
\text { the KWS dat } \\
\text { from [1, } \\
\text { from [1, } \\
\text { the light } \\
\text { the light } \\
\text { the light }\end{array}$ & $\begin{array}{l}\text { ascensi } \\
\text { nation ( } \\
\text { D databa } \\
\text { SIMBAD. } \\
\text { m SIMBAD } \\
\text { day) [4, } \\
\text { atabase } \\
2] . \\
2] . \\
\text { courve } \\
\text { curves } \\
\text { curves }\end{array}$ & $\begin{array}{l}\text { (ICRS) } \\
\text { (ICRS) at } \\
\text { se [3] fo } \\
5,6] . \\
\text { [7] for t } \\
\text { (LC) and } \\
\text { (LC) with } \\
\text { (LC) with }\end{array}$ & $\begin{array}{l}\text { magn } \\
\text { filte } \\
\text { year }\end{array}$ & $\begin{array}{l}\text { ject. } \\
\text { itude with } \\
\text { red and bi } \\
\text { mean magni }\end{array}$ & $\begin{array}{l}\text { galculat } \\
\text { data } \\
\text { de and }\end{array}$ & $\begin{array}{l}\text { ted JD (V } \\
\text { (FBD) and } \\
\text { error of }\end{array}$ & $\begin{array}{l}\text { data) fro } \\
\text { v magnitu } \\
\text { data (YED }\end{array}$ & $\begin{array}{l}\text { om this sit } \\
\text { ude (FB-dat } \\
\text { D) from th }\end{array}$ & $\begin{array}{l}\text { te. } \\
\text { as from site }(Y\end{array}$ & $\begin{array}{l}\text { this site. } \\
\text { YE-data). }\end{array}$ \\
\hline $\mathrm{N}$ & RAJ (2000.0) & DEJ & $(2000.0)$ & SIMBAD & V_mag & Sp_Type & P_rot & KWs & Cycle-1 & Cycle-2 & LC (KWS) & LC (FBD) & LC (YED) & Note \\
\hline 1 & 2 & & 3 & 4 & 5 & 6 & 7 & 8 & 9 & 10 & 11 & 12 & 13 & 14 \\
\hline 1 & 001841.8674 & -080 & 310.806 & HD 1461 & 6.47 & G3VFe0.5 & - & HD 1461 & - & - & LCV-data & LC FB-data & LC YE-data & $\mathrm{C} 4$ \\
\hline 2 & 002251.7883 & -121 & 1233.972 & HD 1835 & 6.39 & $\mathrm{G} 2.5 \mathrm{~V}$ & 8 & $\underline{\text { HD } 1835}$ & $9.1 \pm 0.3$ & Long & $\underline{\mathrm{LC}} \mathrm{V}$-data & $\underline{\text { LC FB-data }}$ & LC YE-data & $\mathrm{C} \sim 6-14, * *, \mathrm{X}$ \\
\hline 3 & 003532.8335 & -003 & 3020.198 & HD 3229 & 5.94 & F5V & 2 & HD 3229 & $4.9 \pm 0.1$ & - & $\underline{\mathrm{LC}} \underline{\mathrm{V} \text {-data }}$ & LC FB-data & LC YE-data & $\underline{\mathrm{P} 1}, \underline{\mathrm{P} 3}, \underline{\mathrm{F}}, \mathrm{C} 1.64, \mathrm{X}$ \\
\hline 4 & 004528.6875 & -125 & 250.912 & $\underline{\mathrm{HD}} 4307$ & 6.15 & G0V & - & $\underline{\mathrm{HD}} 4307$ & - & - & $\underline{\mathrm{LC}} \underline{\mathrm{V} \text {-data }}$ & $\underline{\text { LC FB-data }}$ & LC YE-data & F, P3, P4, C11.23, *** \\
\hline 5 & 011018.7400 & +420 & 3453.309 & HD 6920 & 5.67 & F9IV, F8V & 14 & $\underline{\mathrm{HD}} 6920$ & Var & - & LC V-data & LC FB-data & LC YE-data & C9.48 \\
\hline
\end{tabular}

Рис. 1. Фрагмент HTML-страницы проекта "Вспышки и циклы" с описанием таблицы

Более детально информация о проводимых исследованиях содержится в разделе Cycles $\mathrm{KWS}^{1}$, размещенном на сайте Cycles and Flares².

\section{3 Обработка данных}

Широкоугольные фотометрические наблюдения в проекте KWS выполняются с помощью небольшой ПЗС-матрицы в сочетании с фотообъективом. Это позволяет одновременно наблюдать площадку размером $5^{\circ} \times 7^{\circ} .5$ с возможностью покрытия области до $120^{\circ}$ в течение ночи. Для объектов с блеском в диапазоне 5-11 звездной величины в полосе V достигается фотометрическая точность $5 \%$ или более высокая. Число объектов в базе данных KWS составляет около 200 миллионов.

Для выяснения возможности использования данных KWS в задачах поиска циклической активности были отобраны 72 яркие звезды от $4^{\mathrm{m}} .02$ до $8^{\mathrm{m}} .67$ в полосе $\mathrm{V}$ спектральных типов от $\mathrm{F} 4 \mathrm{~V}$ до $\mathrm{K} 4 \mathrm{~V}$. Для 22 из них с точностью не более 0.5 года были определены возможные циклы магнитной активности (Baliunas et al., 1995; Radick et al., 2018). Отметим при этом, что циклы определялись на основе спектральных наблюдений.

С целью автоматизации обработки данных было написано специальное программное обеспечение, позволяющее исключить из наблюдаемого ряда импульсные события повышения или понижения блеска звезды, что улучшило точность анализа временных рядов. Пример обрабатываемой кривой блеска звезды HD 3229 (F5V, $V=4.9$ ) по данным KWS показан на рис. 2. Из рисунка видно, что интервал наблюдений звезды охватывает промежуток времени около 10 лет.

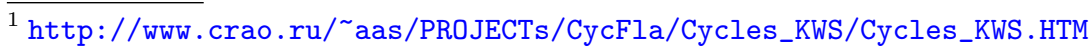

2 http://www.crao.ru/ aas/PROJECTs/CycFla/Cycles_and_Flares.html
} 


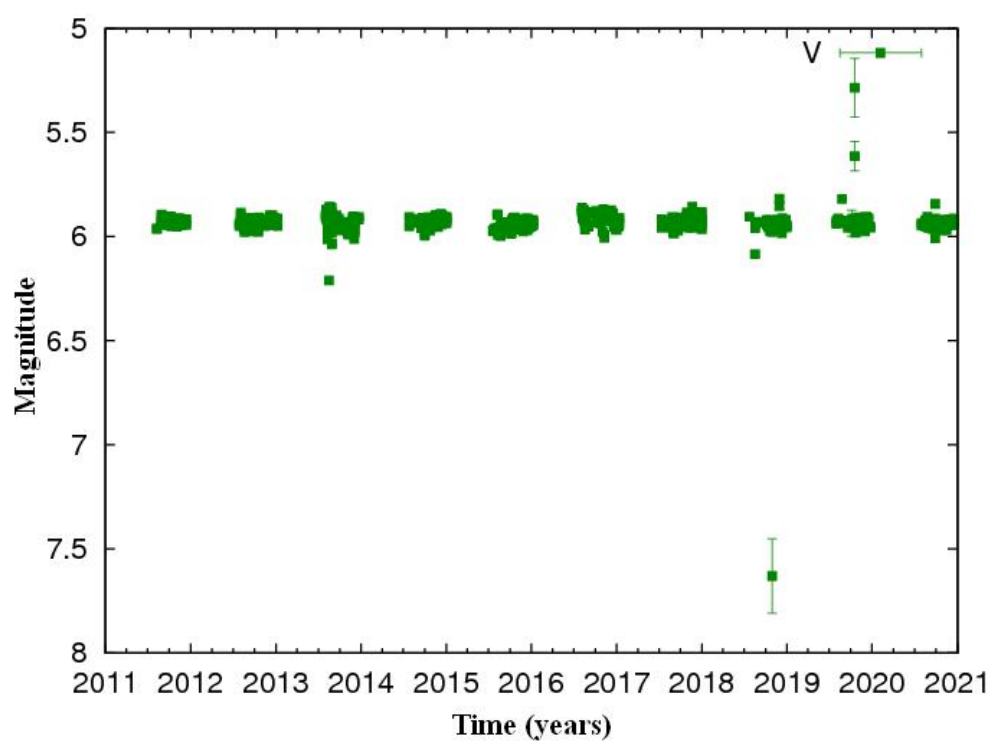

Рис. 2. Оригинальная кривая блеска звезды HD 3229 из базы данных KWS

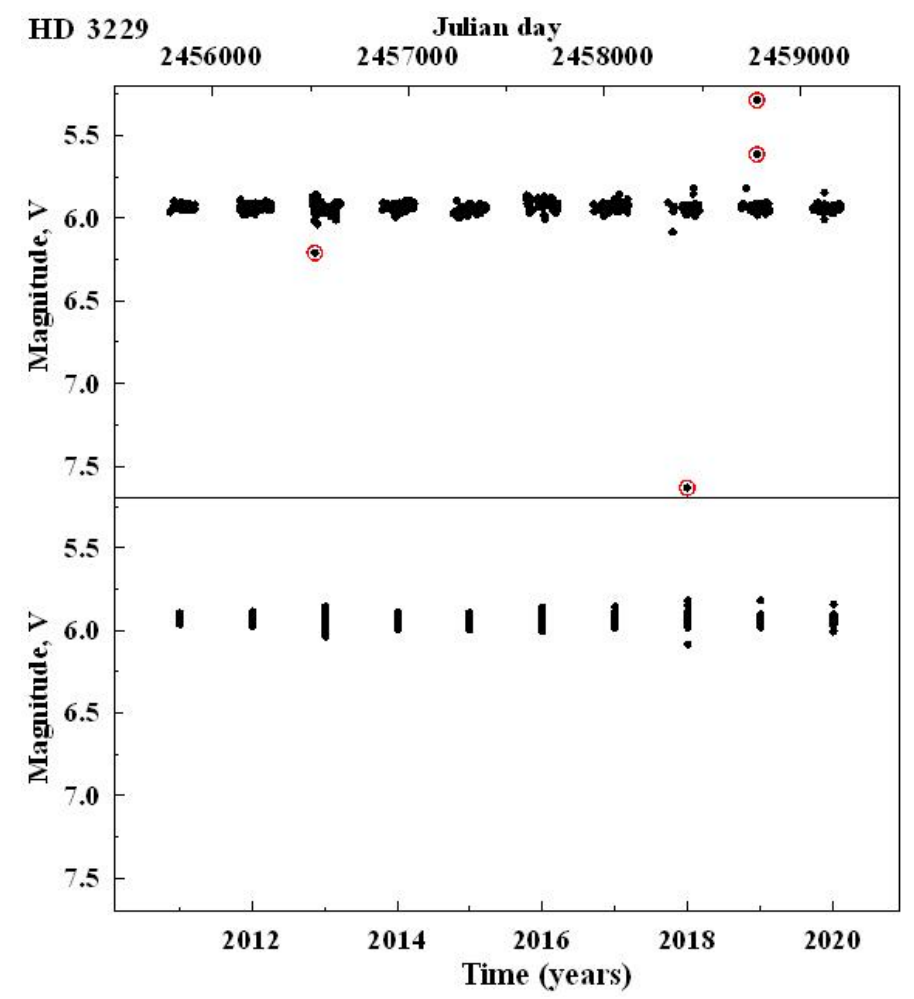

Рис. 3. Оригинальные (верхняя панель) и отфильтрованные (нижняя панель) кривые блеска звезды HD 3229

Рисунок 3 иллюстрирует оригинальные и отфильтрованные кривые блеска исследуемой звезды, в том числе со свёрткой данных по годам (нижняя панель). На верхней панели красными кружками обозначены данные, которые были исключены из рассмотрения. 


\section{4 Анализ на периодичность и возможные циклы}

Результаты периодограммного анализа и свёртки данных с найденными периодами представлены на рисунках 4 и 5. Отметим, что у рассматриваемой звезды мы нашли два возможных периода

HD 3229, Fourier $(\mathrm{F}=0.0016683, \mathrm{~A}=0.0118688)$

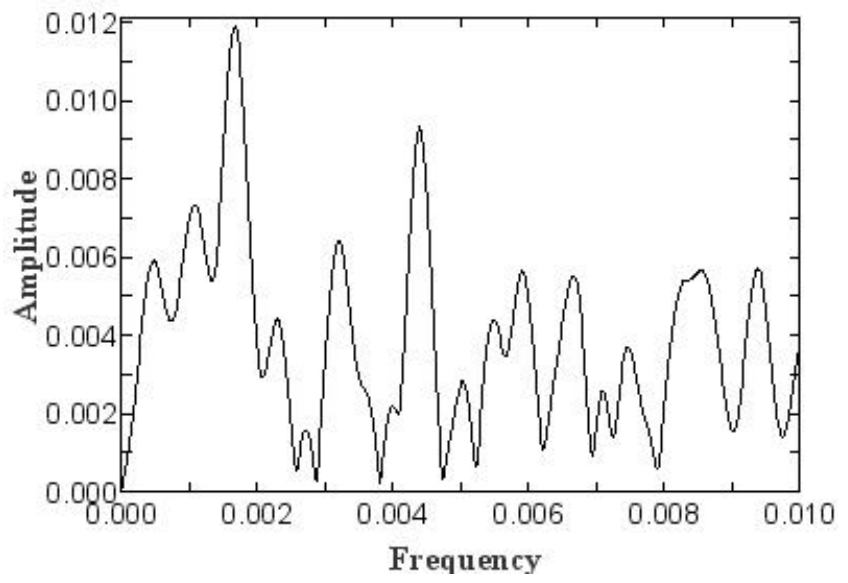

Рис. 4. Результаты периодограммного анализа

циклических изменений блеска: 1.64 г. (рис. 5а) и 4.9 г. (рис. 5б). Соотношение периодов составляет $1 / 3$. С учетом ошибок измерений длительный период соответствует утроенному короткому периоду. Ранее по изменениям индекса хромосферной активности был найден период $4.9 \pm 0.1$ год (Baliunas et al., 1995).

HD 3229, Frequency: 0.0016683 , Pcyc $\sim 1.64$ year

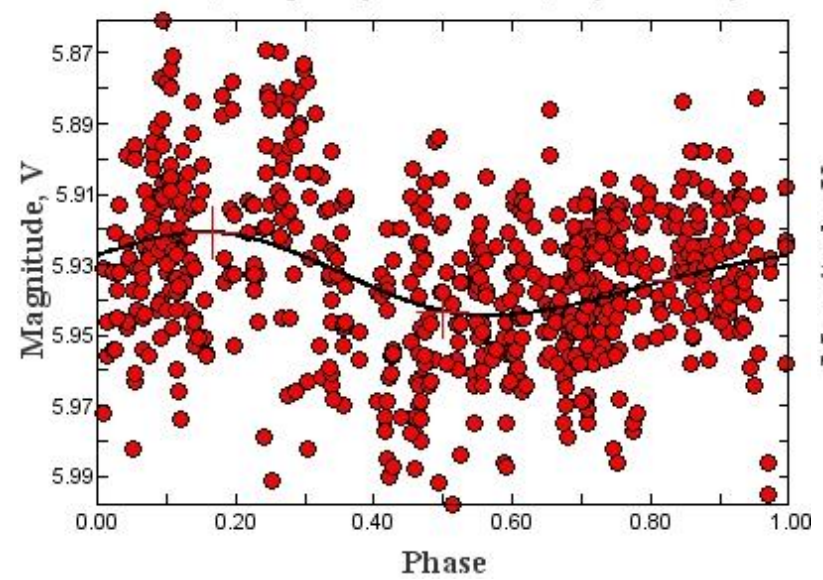

a)

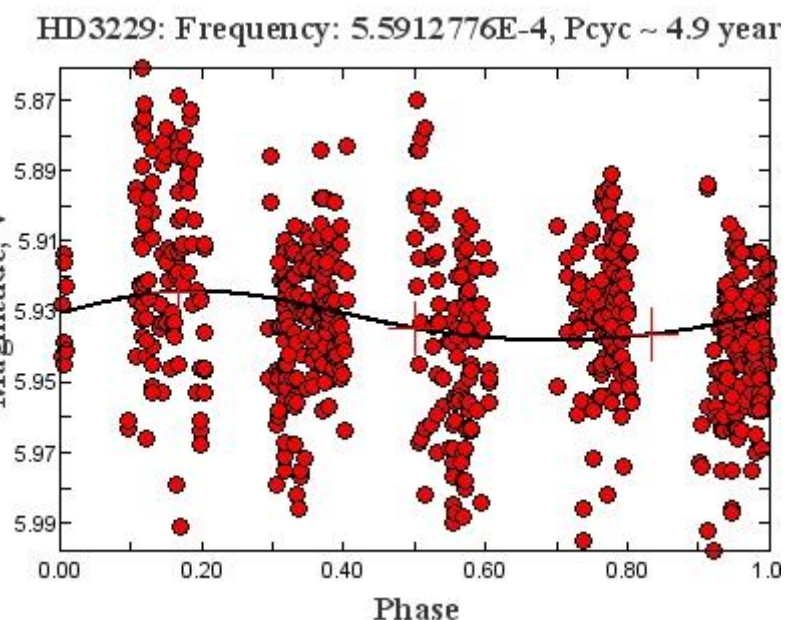

б)

Рис. 5. Возможные циклы изменений блеска звезды HD3229 по данным KWS

\section{5 Заключение}

На примере анализа наблюдений звезды HD 3229 показана возможность использования данных проекта KWS для поиска долговременных малоамплитудных изменений блеска, возможно связанных с циклической активностью. Один из определенных периодов совпадает в пределах ошибок с ранее 
опубликованным значением, полученным в результате анализа многолетних спектральных наблюдений.

Для части звезд выборки возможные циклы активности были определены впервые. Некоторые из ранее определенных циклов не нашли своего подтверждения по результатам обработки использованных нами наблюдений.

На указанном в разделе 2 сайте приведены оригинальные и отфильтрованные кривые блеска исследованных звезд, результаты периодограммного анализа и свёртки данных с найденными периодами.

При выполнении работы активно использовались поддерживаемые Центром астрономических данных в Страсбурге приложения SIMBAD, VizieR и ALADIN, а также библиографический сервис SAO/NASA ADS. Авторы признательны всем, кто обеспечивает их работу. Выполнение работы частично поддержано грантом РФФИ № 19-02-00191.

\section{Литература}

Baliunas S.L., Donahue R.A., Soon W.H., et al., 1995. Astrophys. J., vol. 438, p. 269.

Bondar' N.I., Gorbunov M.A., Shlyapnikov A.A., 2019. Physics of Magnetic Stars. ASP Conf. Ser., vol. 518, p. 180 .

Bondar' N.I., Katsova M.M., 2020. Geomagnetism and Aeronomy, vol. 60, p. 942.

Maehara H., 2014. Journ. Space Sci. Inf. Japan, vol. 3, p. 119.

Radick R.R., Lockwood G.W., Henry G.W., et al., 2018. Astrophys. J., vol. 855, iss. 2, id. 75.

Stepanov R., Bondar' N.I., Katsova M.M., et al., 2020. Mon. Not. Roy. Astron. Soc., vol. 495, iss. 4, p. 3788 .

Wenger M., Ochsenbein F., Egret D., et al., 2000. Astron. Astrophys. Suppl. Ser., vol. 143, p. 9. 


\title{
A search for activity cycles on the selected F, G and $\mathrm{K}$ dwarfs from long-term wide-field photometric observations
}

\author{
A.A. Shlyapnikov, N.I. Bondar', M.A. Gorbunov \\ Crimean Astrophysical Observatory, Nauchny 298409 \\ aas@craocrimea.ru
}

\begin{abstract}
We consider the possibilities of using data from the KWS ground-based panoramic observation project to search for activity cycles on the bright $\mathrm{F}-\mathrm{K}$ dwarfs. Methods and results of processing observational data are presented, and the formation of an interactive database is described.
\end{abstract}

Key words: solar-type stars, red dwarfs, activity cycles, databases 